\title{
Analysis of Finnish blue mussel (Mytilus edulis L.) shell: Biomineral ultrastructure, organic-rich interfacial matrix and mechanical behavior
}

\author{
Pezhman Mohammadi ${ }^{a,}{ }^{,}$, Wolfgang Wagermaier ${ }^{b}$, Merja Penttila ${ }^{a}$ and Markus B. Linder ${ }^{c, *}$
}

\author{
aVTT Technical Research Centre of Finland Ltd., Espoo, FI-02044, VTT, Finland \\ ${ }^{b}$ Department of Biomaterials, Max Planck Institute of Colloids and Interfaces, D 14424 Potsdam (Germany) \\ 'Department of Bioproducts and Biosystems, School of Chemical Engineering, Aalto University, P.O. Box 16100, FI-16100, \\ Espoo (Finland)
}

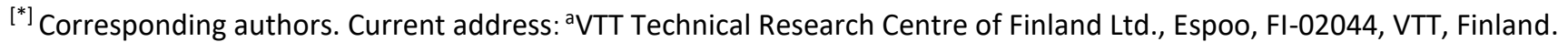
Department of Bioproducts and Biosystems, School of Chemical Engineering, Aalto University, P.O. Box 16100, FI-16100, Espoo (Finland). E-mail address: pezhman.mohammadi@vtt.fi and markus.linder@aalto.fi
\end{abstract}

Key words: Finnish blue mussel; Biomineralization; Interfacial matrix protein; Chitin

\begin{abstract}
Studying various marine biomineralized ultrastructures reveals the appearance of common architectural designs and building blocks in materials with fascinating mechanical properties that match perfectly to their biological tasks. Advanced mechanical properties of biological materials are attributed to evolutionary optimized molecular architectures and structural hierarchy. One example which has not yet been structurally investigated in great detail is the shell of Mytilus edulis L. (Finnish blue mussel) found in the archipelago of SW-Finland. Through a combination of various state-of-the-art techniques such as high-resolution electron microscopy imaging, Fourier-transformed infrared spectroscopy, powder X-ray diffraction, synchrotron wide-angle X-ray diffraction, nanoindentation and protein analysis, both the inorganic mineralized components as well as the organic-rich matrix were extensively characterized. We found very similar ultra-architecture across the shell of M. edulis L. as compared to the widely studied and closely related M. edulis. However, we also found interesting differences, for instance in the thickness and degree of orientation of the mineralized layers indicating dissimilar properties and related alterations in the biomineralization processes. Our results show that the shell of M. edulis L. has a gradient of mechanical properties, with the increase in the stiffness and the hardness from anterior to the posterior region of the shell. The shell is made from distinct and recognizable mineralized layers each varying in thickness and microstructural features. At posterior regions of the shell, moving from dorsal to ventral side, these layers are an oblique prismatic layer, a prismatic layer and a nacreous layer, in
\end{abstract}


which the oblique prismatic layer is found to be the main and thickest mineralized layer of the shell. Probing the calcified rods in the oblique prismatic layer using high resolution SEM imaging revealed opening of channels with a diameters of $40-50 \mathrm{~nm}$ and lengths up to a micrometer extending through the rods. The chitin and protein have been found to be the main component of the organic-rich interfacial matrix as expected. Protein analysis showed two abundant proteins with sizes around $100 \mathrm{kD}$ and $45 \mathrm{kD}$ which likely not only regulates biomineralization and adhesion of the crystals but also governing the intrinsic-extrinsic toughening in the shell. Overall, this detailed analysis provides new structural insights into biomineralization of marine shells in general.

\section{Introduction}

Biological materials show fascinating properties and in many ways can outperform man-made engineered materials, although they are built from very limited elements appearing in nature and comparable simple building blocks ${ }^{1}$. Exceptional examples of such materials can be found in marine biomineral structures that combine high stiffness, strength, and toughness. Such materials achieve their function by assembling large fractions of anisotropic inorganic stiff and strong elements, embedded in an isotropic soft and energy-dissipating adhesive organic matrix ${ }^{2}$. These two basic components with mismatching properties are merged in a complex hierarchical architecture, which ultimately enables absorption of energy at different length scales. This is driven by evolutionary optimized molecular interactions among various building blocks throughout the course of the evolution.

For instance, nacre in the interior of mollusk shells -also known as mother of pearl- features a brick and mortar microstructure, assembled from 0.4-0.5 $\mu \mathrm{m}$ thick and 5-15 $\mu \mathrm{m}$ wide tablets of calcium carbonate (aragonite) that are glued to one another with few nanometer thin continuous layers of adhesive and energy dissipating organic matrix ${ }^{3,4}$. Orders of magnitude tougher than nacre, conch shell is another example of a marine biomineral structure with a three-tier multiscale lamellar structure ${ }^{5-7}$. Shells consist of outer, middle and inner layers, each further constitute of first ( $5 \mu \mathrm{m}$ thick and several $\mu \mathrm{m}$ wide), second (5-30 $\mu \mathrm{m}$ thick and 5-60 $\mu \mathrm{m}$ wide) and third (60-100 nm thick and 100-400 nm wide) order lamellae varying in thickness and length of the aragonite platelets that are glued to one another by the organic matrix. This hierarchical, lamellar ultra-architecture provides various crack deflecting pathways, bridging and fiber pullout, hence increasing the toughness.

Details of assembly mechanisms and the formation of such complex hierarchical structures are still under investigation. However there is growing evidence illustrating that the organic-rich interface consisting 
of silk-like and acidic proteins along with a chitin network ${ }^{8,9}$, largely governs both nucleation and inhibition of the crystal growth and providing a matrix structure that has substantial importance toward structural integrity of the shell ${ }^{10,11}$.

In recent years, biological materials have been studied to understand basic principles of biomineralization making those available for material synthesis as well as one main source of inspiration for fabrication of next generation high performance and advanced materials (Studart, 2016, 2012; Wegst et al., 2015; Xu et al., 2007). These potential applications range from advanced medical devices, robust electronics apparatus, and sensors, ${ }^{15-17}$ to lightweight aerospace application and military armors ${ }^{18,19}$.

Despite its scientific, ecological and economic importance, the current understanding of such complex mineral producing machinery is far from complete. This motivates to analyze the ultrastructure and mechanical properties of biomineralized materials which have never been studied before to identify common or dissimilar architectural features and shade light onto outstanding mechanical characteristics of such materials. Furthermore, a fundamental understanding of structure-property relations has substantial implications for design strategies and manufacturing processes of next-generation advanced functional materials ${ }^{20}$.

In this study, we provide extensive characterization of the shell of M. edulis L. (Finnish blue mussel) found in the archipelago of SW-Finland (Northern Baltic Sea) for the first time. This was carried out by combing various state of the art techniques to study both inorganic mineralized components as well as the organic matrix, including high-resolution electron microscopy imaging, Fourier-transform infrared spectroscopy (FTIR), RAMAN-spectroscopy, powder X-ray diffraction, synchrotron wide-angle X-ray diffraction (WAXS), Nanoindentation and proteomic analysis.

\section{Method and materials}

\subsection{Research specimens}

Live specimens of $M$. edulis L. were collected from the archipelago of SW-Finland in the northern Baltic Sea. Shells were dissected and removed. The outer sides of the shells were cleaned mechanically for removing contaminants and epibionts. The inner sides of the shells were also cleaned from connective tissue and rinsed with cold $\left(4^{\circ} \mathrm{C}\right)$ water to remove any loose organic debris. Samples were immediately flash-frozen in liquid nitrogen and kept at $-80^{\circ} \mathrm{C}$ in a freezer until use. 


\subsection{Scanning electron microscopy (SEM)}

SEM imaging carried out with a Zeiss FE-SEM field emission microscope (Microscopy center, Aalto University, Espoo, Finland) with variable pressure, operating at $1-1.5 \mathrm{kV}$ operating in low vacuum mode for imaging hydrated specimens.

\subsection{Powder X-ray diffraction (XRD)}

XRD carried out using Rigaku SmartLab, equipped with HyPix-3000 Hybrid Pixel Array Detector. Collection performed using $\mathrm{CuK} \alpha$-radiation of $\lambda=1.5418 \AA$ (energy of $45 \mathrm{kV}$ and $40 \mathrm{~mA}$ ). The diffractometer collected at a $2 \theta$ range of $10-80^{\circ}$ with the step size of $0.01 \%$ and 4 sec exposure time. The crystalline phases were identified by matching the XRD patterns with the Joint Committee on Powder Diffraction Standards (JCPDS) database.

\subsection{Synchrotron wide-angle $\mathrm{X}$-ray scattering (WAXS) measurement}

Wide-angle X-ray diffraction experiments were performed at the $\mu$ Spot beamline at BESSY II synchrotron source (Helmholtz-Zentrum Berlin für Materialien und Energie, Germany). The measurements were carried out using a silicon (111) monochromator with an X-ray wavelength of $0.82656 \AA$ (energy of $15 \mathrm{keV}$ ) and a beam size of $50 \mu \mathrm{m}$. The beamline calibration was done with a quartz $\left(\mathrm{SiO}_{4}\right)$ standard giving a sample to detector distance of approximately $280 \mathrm{~mm}$. Diffraction patterns were collected using a two-dimensional CCD detector (MarMosaic 225, Mar USA, Evanston USA) with a pixel size of $73.242 \mu \mathrm{m}$ and $3072 \times 3072$ pixels. Intensities have been characterized using the software DPDAK after subtraction of air scattering from the diffractogram ${ }^{21}$.

\subsection{Nanoindentation}

Nanoindentation was performed under ambient conditions using an Ubi nanoindentation instrument (Hysitron) with a Berkovich diamond tip. To obtain reduced modulus and hardness, the loaddisplacement curves were analyzed using the methods described by Oliver and Pharr. ${ }^{22}$ More than 1000 indentations were performed per sample using a maximum load of $1500 \mu \mathrm{N}$ on the polished surface. At peak load, a dwell time of $10 \mathrm{~s}$ was applied to account for creep behavior. At five different regions across each sample, two adjoined lines were measured with steps of $15 \mu \mathrm{m}$ (between lines) and $6.2 \mu \mathrm{m}$ between each indent along the line. Therefore, plastically deformed zones from previous indents did not affect the measurements. The effect of the topography was minimized by finely polishing the sample surface. 


\subsection{Fourier transform infrared spectroscopy (FTIR)}

Infrared measurement carried out using Unicam Mattson 3000 FTIR spectrometer directly on the finely powdered shell. To do that, the specimen was mixed with potassium bromide (1:9 mixing ratio of the specimen in $\mathrm{KBr}$ ) which was then turned into $1 \mathrm{~mm}$ transparent discs under high pressure and measured in transmission mode. All spectra were scanned within the range of $400-4000 \mathrm{~cm}^{-1}$, with a total of 32 scans and a resolution of $32 \mathrm{~cm}^{-1}$.

\subsection{Protein extraction}

Shells were incubated with $1 \%, \mathrm{v} / \mathrm{v} \mathrm{NaOCl}$ for 24 hours at $4{ }^{\circ} \mathrm{C}$ in order to remove superficial organic contaminants as well as the periostracum. Shells were then rinsed with deionized water and dried at ambient temperature before mechanically crushing them into powder in a cold room $\left(4{ }^{\circ} \mathrm{C}\right)$. Decalcification carried out by addition of $50 \mathrm{ml} 1 \mathrm{M}$ EDTA pH 8 to every $5 \mathrm{~g}$ of shell powder for 24 hours at $4{ }^{\circ} \mathrm{C}$ and centrifuged at $40000 \mathrm{~g}$ for $60 \mathrm{~min}$. Supernatants collected and dialyzed against MQ water and then freeze-dried. Pellets from each method also rinsed three times with MQ water and freezedried. Extracted proteins separated and analyzed with standard sodium dodecyl sulfate-polyacrylamide gel electrophoresis (SDS-PAGE) ${ }^{23}$.

\subsection{MALDI-TOF-TOF}

Proteins identity was confirmed by matrix-assisted laser desorption/ionization-time of flight/time of flight (MALDI-TOF-TOF) mass spectrometer (UltrafleXtream ${ }^{\mathrm{TM}}$ Bruker, Aalto department of biotechnology and chemical technology facilities, Espoo, Finland) equipped with a 200-Hz smart-beam laser (337 nm, 4 ns pulse). For partial peptide sequencing (MS/MS), in-gel tryptic digestion performed using proteoprofile trypsin in-gel digest kit (PP0100, Sigma-Aldrich). The samples were desalted using either ZipTip ${ }^{\mathrm{TM}} \mathrm{C} 18$ (Millipore) eluted directly onto MALDI target plate using MALDI matrix (a-cyano4-hydroxycinnamic acid, $10 \mathrm{mg} / \mathrm{ml}$ in $70 \% \mathrm{ACN}, 0.03 \% \mathrm{TFA}$ ). Spectra acquired in the reflection positive ion mode and resulting monoisotopic masses cross-referenced against the database (NCBI) with a tolerance of $150 \mathrm{ppm}$. Protein identification performed using the MASCOT search engine (Matrix Science, London, UK; version 2.1) against NCBI server (http:// www.ncbi.nlm.nih.gov). The peptide mass and fragment ion tolerances were set to 0.5 Da for MS/MS data searched. The peptide peaks further 
bioRxiv preprint doi: https://doi.org/10.1101/636696; this version posted August 12,2019 . The copyright holder for this preprint (which was not certified by peer review) is the author/funder, who has granted bioRxiv a license to display the preprint in perpetuity. It is made available under aCC-BY 4.0 International license.

manually investigated by the interpretation of the raw MS/MS spectra to perform de novo sequences. Furthermore resulted peptides cross-referenced against BLASTp, Swiss-Prot, and UniProt databases. 
(A)

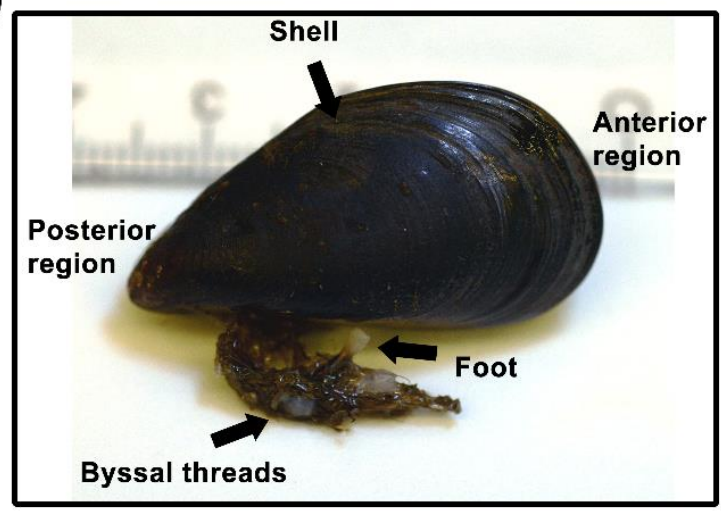

(C)

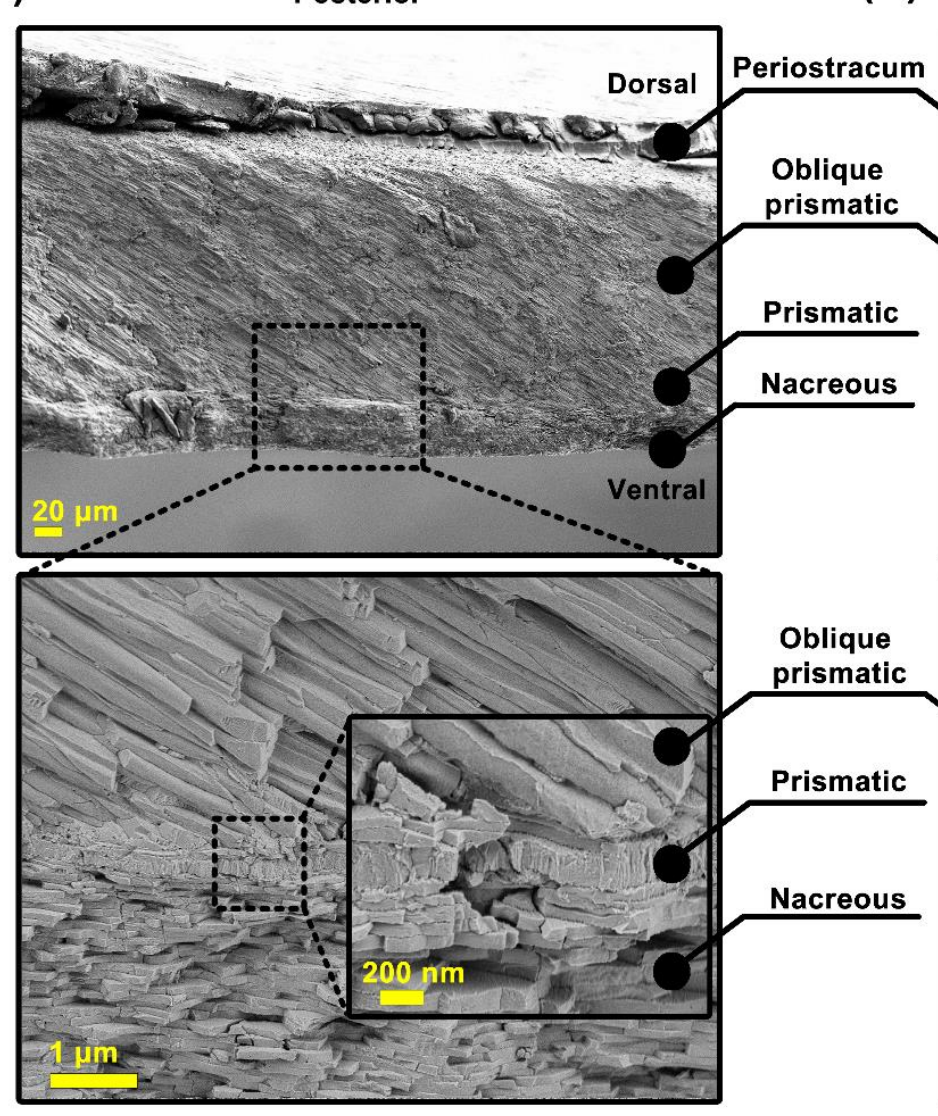

(B)

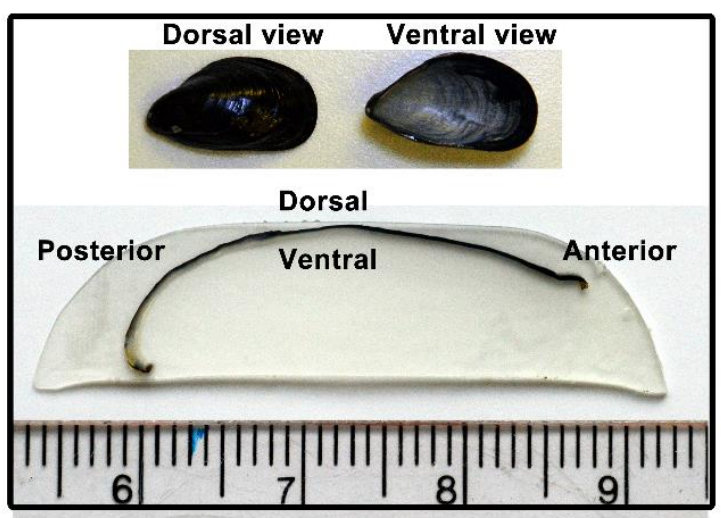

(D)

Anterior
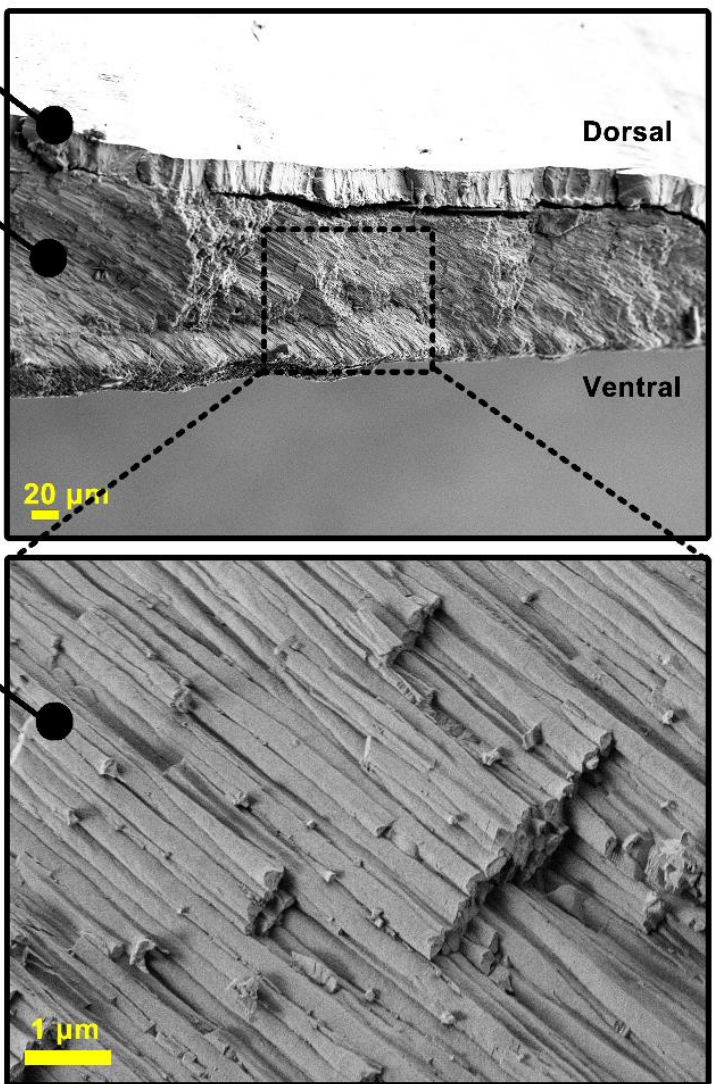

Fig. 1. (A) Morphological features of an adult M. edulis L. shell, commonly known as Finish blue mussel obtained from the archipelago of SW-Finland in the northern Baltic Sea. (B) Longitudinal cross-section of the shell. (C and D) SEM micrographs from the cross-section of the shell taken from posterior and anterior regions at different magnifications. SEM images illustrating periostracum layer, oblique prismatic layer, prismatic layer and a nacreous layer which can be found at the posterior region. At the anterior region, only periostracum and oblique prismatic layers can be found. 


\section{Results and discussion}

\subsection{Research specimen}

Selected M. edulis L. obtained from the archipelago of SW-Finland in the northern Baltic Sea for this study had a size of about 2.5-3 cm (anterior-posterior length) (Fig. 1A, B, and S1). In general, the size of the adult $M$. edulis L. is relatively smaller than other extensively studied and closely related taxa of mussels such M. edulis (commonly found in North Atlantic and Pacific), M. galloprovincialis (Mediterranean, black sea, and North Pacific) and M. trossulus (North Atlantic and Baltic Sea). Mytilus specimens in our study have been found to be a crossbreed between M. edulis and M. trossulus ${ }^{24}$.

\subsection{Biomineral ultra-architecture of the shell}

Scanning electron microscopy (SEM) of cross-sections of the shell revealed presence of multiple layers, however distinct and recognizable mineralized layers each varying in thickness and microstructural features. At the posterior regions of the shell, from dorsal to ventral side, the outer most layer starts with an organic layer called periostracum. Periostracum is a protective leathery layer that has a substantial importance for the mineralization and templating of the first calcified layer. Subjacent to the periostracum is the calcified oblique prismatic layer, exhibiting a closely packed, ordered and highly elongated rodlike ultrastructure, which is developed around $19^{\circ}$ relative to the periostracum layer. Subsequently, the prismatic and nacreous layers are located, both developed perpendicular to the periostracum (Fig. 1C). The prismatic layer consists of radially elongated short monocrystals while the interior nacreous layer comprises a brick and mortar microstructure, assembled from tablets with uniform thickness and widths. Among all, oblique prismatic sheets represent the main and thickest $(\simeq 200 \mu \mathrm{m})$ mineralized layer. The oblique prismatic layer alone constitutes about $86 \%$ of the overall shell thickness in comparison to the nacreous $(\simeq 30 \mu \mathrm{m})$ and prismatic $(\simeq 0.4 \mu \mathrm{m})$ layer which only represent about $13 \%$ and less than 0.05 $\%$ respectively. In addition, probing the cross-section of the shell at the anterior region (growth front) revealed the presence of only the periostracum and oblique prismatic layer (with a thickness of $\simeq 125 \mu \mathrm{m}$ forming $100 \%$ calcified layer) (Fig. 1D). We found many similarities in the ultra-architecture of the $M$. edulis L. shell in this study and the closely related M. edulis. However, we also found differences in the thickness of the layers comparing these result to an earlier studied adult M. edulis shells ${ }^{25}$. Table 1 summarizes these differences. 


\begin{tabular}{|c|c|c|c|c|}
\hline \multicolumn{5}{|c|}{ Table 1. M. edulis L. versus M. edulis shell } \\
\hline \multirow{2}{*}{ Layers } & \multicolumn{2}{|c|}{ M. edulis L. } & \multicolumn{2}{c|}{ Mytilus edulis } \\
\cline { 2 - 5 } & $\mu \mathrm{m}$ & $\%$ & $\mu \mathrm{m}$ & $\%$ \\
\hline Inorganic & \multicolumn{5}{|c|}{} \\
\hline Oblique prismatic & 200 & 86 & 296 & 80 \\
\hline Prismatic & 0.2 & 0.05 & 4 & 1 \\
\hline Nacreous & 30 & 13 & 66 & 18 \\
\hline Organic & & & & \\
\hline Periostracum & 30 & - & 15 & - \\
\hline
\end{tabular}

Table 1. Comparison between the thickness of inorganic and organic layers in the M. edulis L. and M. edulis shell.

\subsection{Calcite and aragonite}

To investigate the nature of the shell and its variability in the calcified composition we performed XRD. The results (Fig. 2A) illustrated that the shell, in fact, contains calcium carbonate $\left(\mathrm{CaCO}_{3}\right)$ which has been shown for many other seashells ${ }^{26}$. Spectra showed that the shell mainly constitutes of calcite, but reflections corresponding to aragonite were also noticeable. Calcite and aragonite both were found to be the most stable polymorph of $\mathrm{CaCO}_{3}$. We further mapped the distribution of each polymorph across the shell using simultaneous high-resolution synchrotron WAXS/SAXS mapping (Fig. 2B and C). This revealed two very distinct phases of calcite and aragonite. A thick outer region comprises calcite corresponding to the oblique prismatic layer and a thinner region toward the ventral side comprise of aragonite corresponding to prismatic and nacreous layers. 

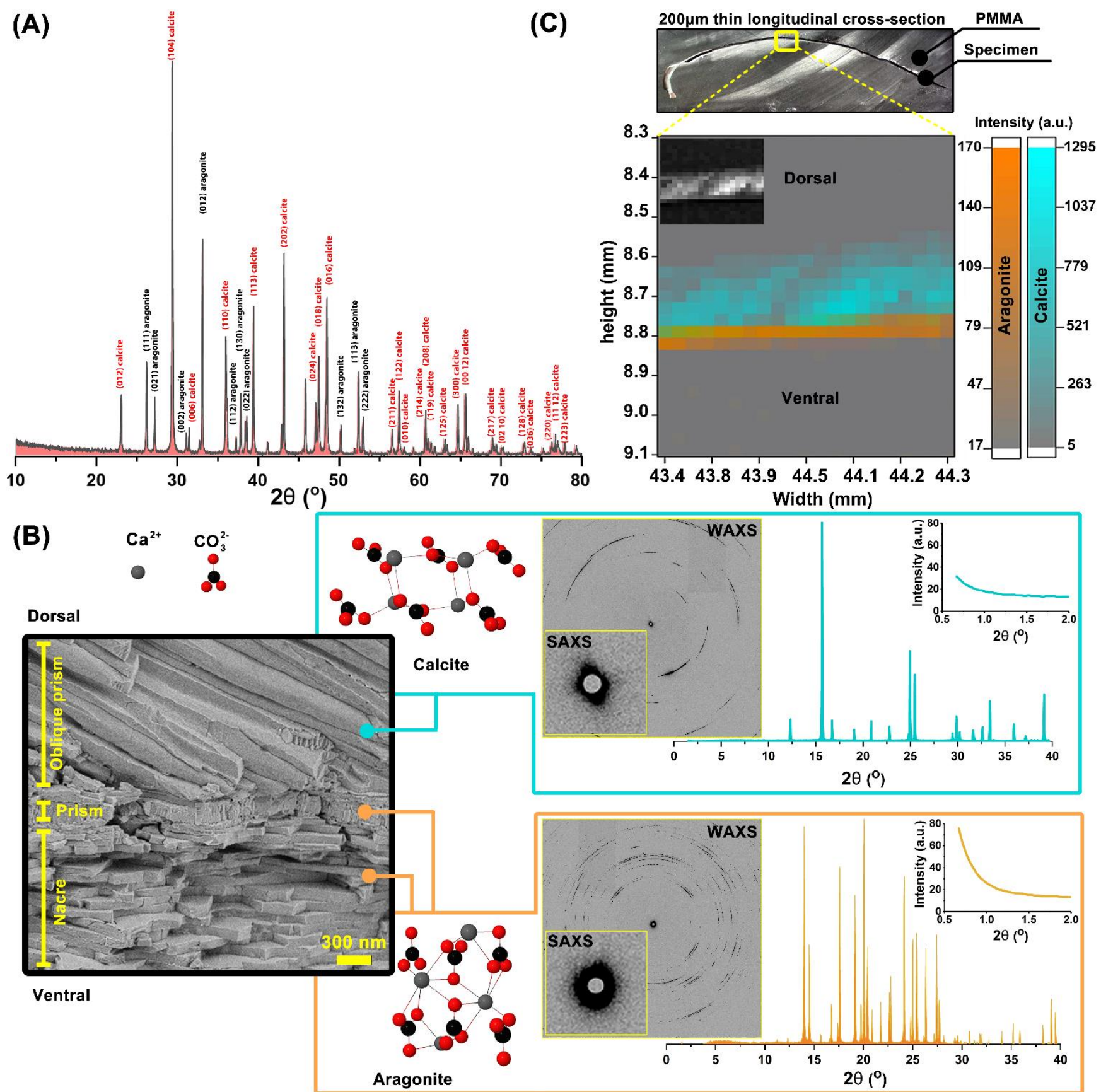

Fig. 2. (A) XRD of the bulk shell powder. (B) Representative $2 D$ synchrotron WAXS/SAXS diffraction patterns and their extracted $1 D$ profile corresponding to oblique prismatic and nacreous mineralized layers. (C) Heat-map illustrating scattering intensity distribution of the calcite and aragonite throughout the ultra-architecture of the shell. Each square corresponds to single measurement point. Cyan indicates distribution of the calcite and orange displays the aragonite. 
bioRxiv preprint doi: https://doi org/10.1101/636696; this version posted Auqust 12, 2019. The copyright holder for this preprint (which was not certified by peer review) is the author/funder, who has granted bioRxiv a license to display the preprint in perpetuity. It is made available under aCC-BY 4.0 International license.

\subsection{Oblique prismatic layer}

High magnification SEM images from oblique prismatic showed that it is made from individual calcified rod-like ultrastructure with diameter of $0.5 \mu \mathrm{m}$ and lengths stretching from 120 to $200 \mu \mathrm{m}$ (aspect ratio ranging from 1:240 to 1:400) in posterior and anterior region respectively. Rods were tightly packed and oriented at $19^{\circ}$ (posterior region) relative to periostracum layer and $22^{\circ}$ (anterior region) (Fig. 1D). An earlier report demonstrated $45^{\circ}$ of orientation for the rods in M. edulis in the anterior region ${ }^{25}$. However, the degree of orientation of the rods in the posterior region of the shell was not in the scope of earlier studies. We further investigated the morphology of the rods in more detail using high-magnification SEM imaging (Fig. 3). We noticed the presence of nanometer-sized pores with a diameters of about 40-50 nm. These were randomly distributed throughout the length of the rods. Investigating cracked rods, we noticed that in fact these pores are opening of channels extending at around $1 \mu \mathrm{m}$ in length within the core of the calcified rods, mostly present at the anterior regions close to the ventral side.

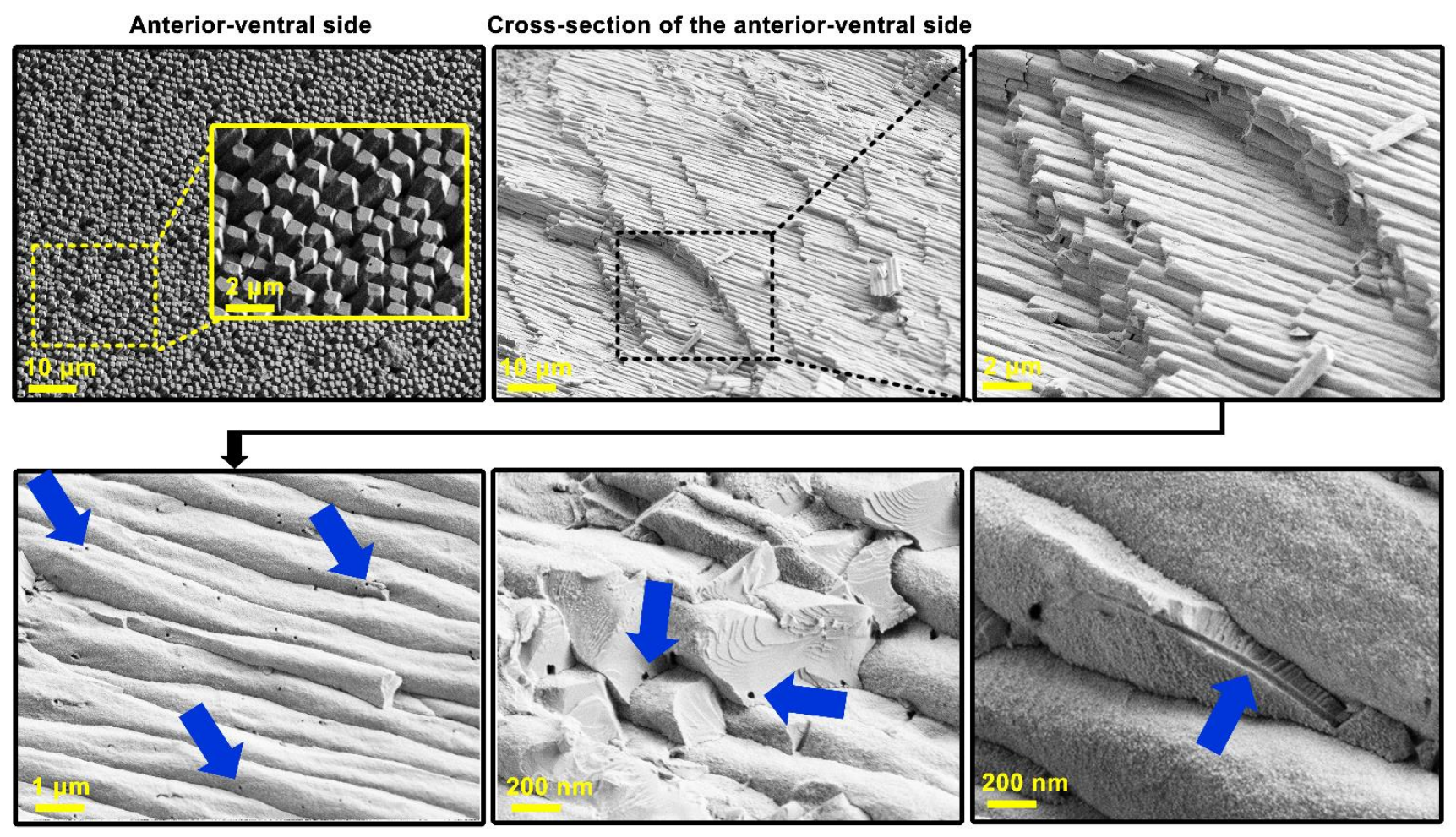

Fig. 3. Characterization of the calcified rods in the oblique prismatic layer located at the anteriorventral side of the shell. High magnification SEM images revealed the presence of pores with diameters of 40-50 nm forming openings of channels that extend through the rods. Blue arrows indicate the opening of the pores. Fracturing the rods also shows also that the channels extended through the rods. 


\subsection{Mechanical properties}

To gain additional insight into the mechanical properties of the shell, we performed nanoindentation mapping on longitudinal and transversal cross-sectional cuts of the two identical shell originating from the same mussel (Fig. 4A, B, and fig. S2A). Probing the longitudinal cut exhibited a gradient of mechanical properties. Moving from anterior to the posterior region we collected the profile at nine positions, each three millimeters apart from each other and noticed an increase in the stiffness value of about $53 \pm 5.9 \mathrm{GPa}$ to $80 \pm 16.5 \mathrm{GPa}$. For the same set of experiment, the calculated hardness showed values increasing from $1.5 \mathrm{GPa}$ at the anterior region to around $4 \mathrm{GPa}$ at the posterior region. We then performed nanoindentation on the other shell, in which we made a transversal cut corresponding to position five of the longitudinal cut and performed nanoindentation at five different positions (Fig. 4C, D, and fig. S2B). Moving laterally, Young's modulus was ranging from 60 to $66 \mathrm{GPa}$ to and hardness value ranging from 2.5 to $3 \mathrm{GPa}$. 
(A)

\section{Longitudinal cross-section}
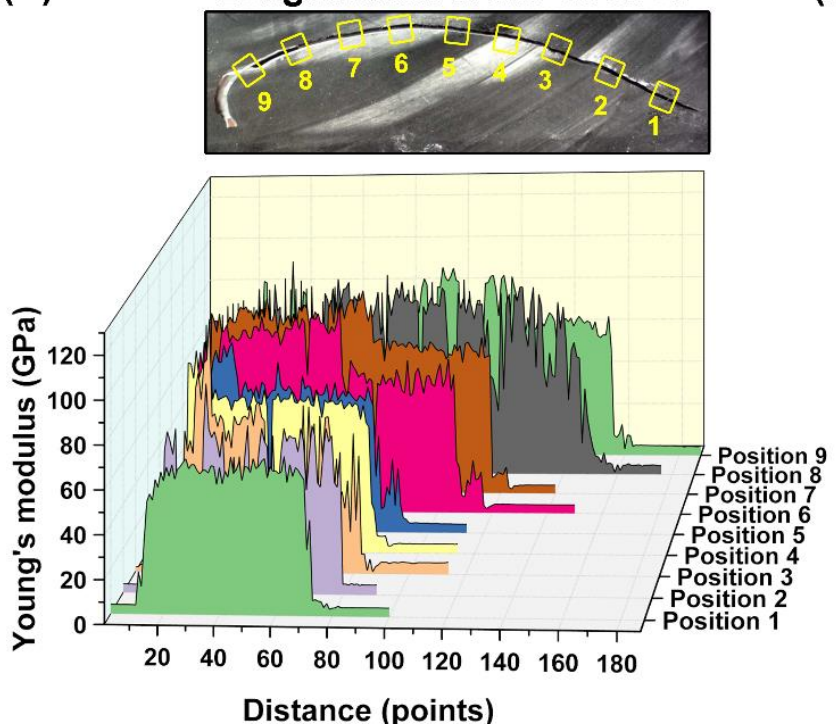

(C)

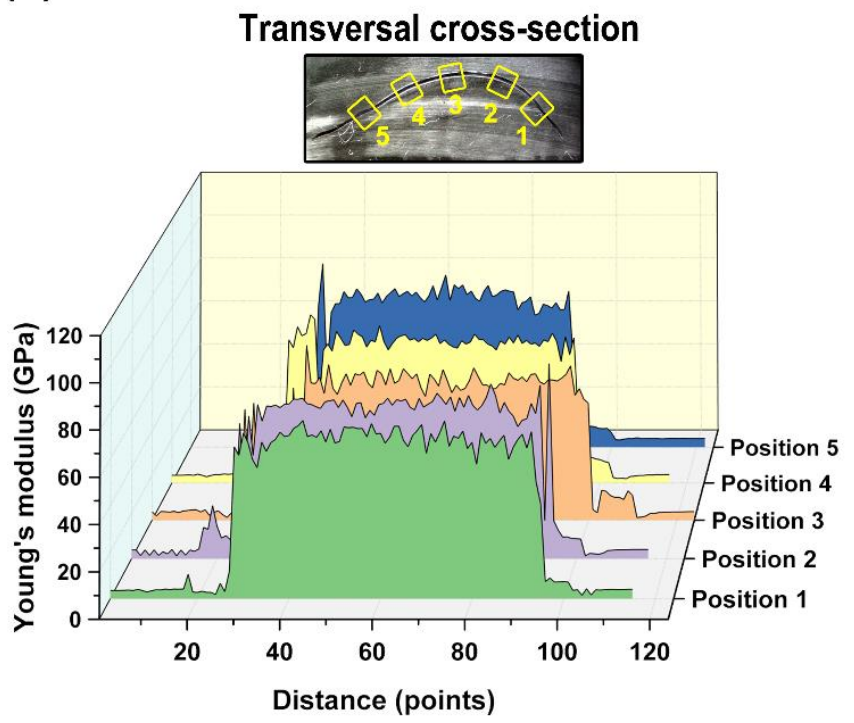

(B)

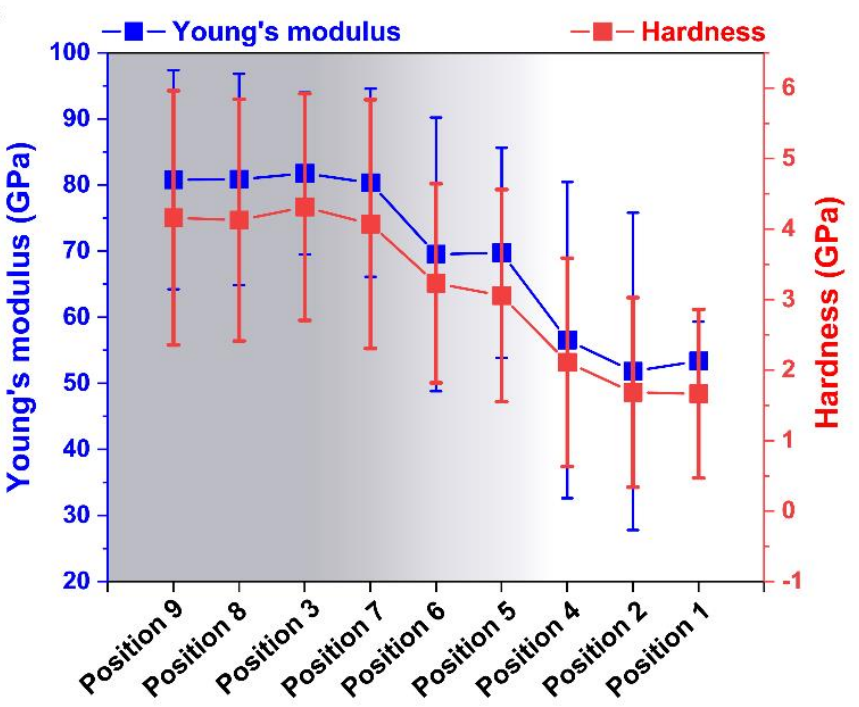

(D)

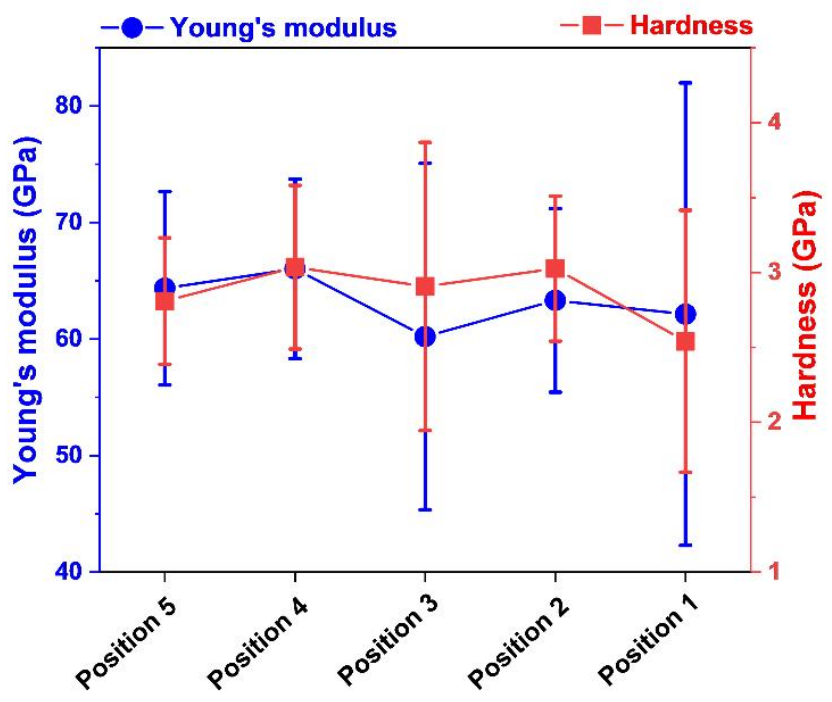

Fig. 4. Mechanical properties derived from nanoindentation experiments performed on the longitudinal and transversal cross-section of the shell. (A) Profile of Young's moduli obtained at nine different positions across longitudinal cross-section and (B) average Young's modulus and hardness values for each position. (C) Profile of Young's moduli obtained at nine different positions across transversal crosssection and $(D)$ average Young's modulus and hardness value for each position.

\subsection{Organic-rich interface}

By studying fracture surfaces of the hydrated oblique prismatic layer, we noticed the presence of an organic adhesive matrix filling the interfaces between the rods (Fig. 4A). Most importantly, we noticed 
that the gel-like adhesive matrix not only oozed out from the interfaces of the rods but also from the openings of the nano-sized pores. Similar gel-like organic matrix have been observed in fractured shell of Atrina rigida and Pinctada margaritifera ${ }^{4}$.This provided the basis to hypothesize that the observed nanosized pores may have a function in transporting matrix material during the biomineralization and adhesion of the rods. In addition, we identified regions in which adhesive organic matrix formed filaments, bridging cracks while fracturing. It is noticeable that filaments are distinctly oriented perpendicular to the direction of the crack (Fig. 4A), suggesting resistance to fracture by dissipating energy through the formation of nano-sized filaments which deflects the cracks into regions in which propagation becomes more difficult.

In order to investigate the nature of the matrix and the compositions in more detail and compare to earlier studies, we performed FTIR experiments in transmission mode. Fig. 5B and C illustrate FTIR spectra of the bulk shell powder, containing soluble and insoluble organic remains after decalcification (either using $1 \mathrm{M}$ EDTA or $5 \% \mathrm{v} / \mathrm{v}$ acetic acid). Spectra strongly exhibit the presence of organic-rich-matrix and calcium carbonate (Table S1). The organic-rich-matrix exhibits three main FTIR band ranges ${ }^{27-29}$. At $834-899 \mathrm{~cm}^{-1}$ stretching vibrations of the C-O-C and C-C bonds corresponded to $\alpha$-chitin as expected. In the range of $1100-1700 \mathrm{~cm}^{-1}$ multiple bands showed up, mainly corresponding to amide (I, II and III) bands of protein $(\mathrm{C}=\mathrm{O}, \mathrm{C}-\mathrm{N}, \mathrm{N}-\mathrm{H}, \mathrm{C}-\mathrm{N}$ and $\mathrm{C}-\mathrm{C}$ vibrations $)$ and $\alpha$-chitin $\left(\mathrm{CH}_{2}, \mathrm{OH}\right.$, and $\mathrm{C}=\mathrm{O}$ vibration $)$. Finally, the $2962 \mathrm{~cm}^{-1}$ band corresponds to amide B band of $\alpha$-chitin. 


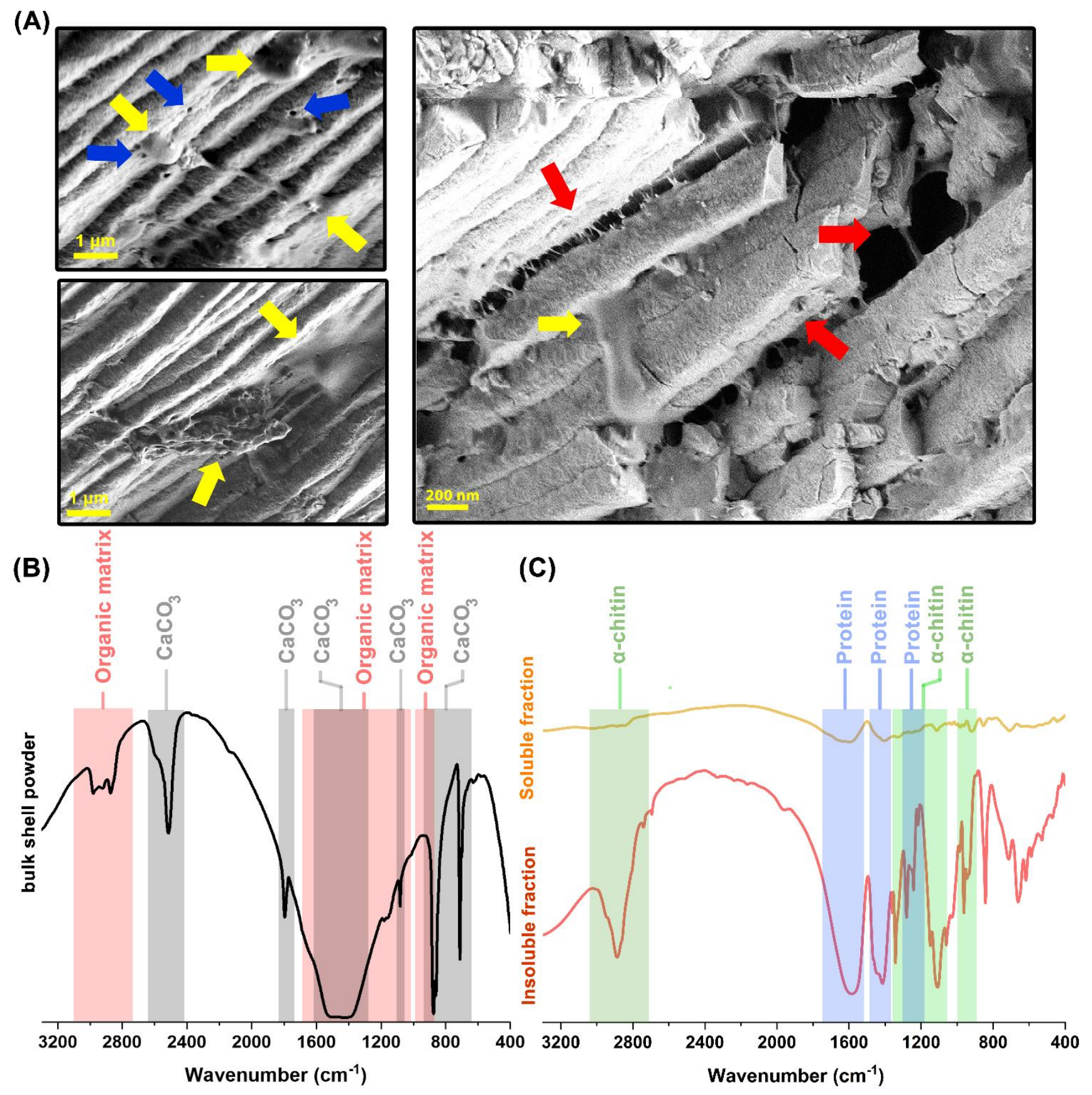

Fig. 5. (A) Environmental SEM images from the fracture surface of the hydrated oblique prismatic layer, illustrating organic adhesive matrix filling the interfaces between the calcified rods also regions in which the organic-rich matrix bridges the crack by forming filaments. Yellow arrows show a gel-like adhesive matrix that oozes out from the interfaces of the rods but also from the openings of nano-sized channels shown by blue arrows. Red arrows show the filaments bridging the cracks and they are oriented 
perpendicular in the direction of crack propagation. (B) FTIR spectra of the bulk shell powder. (C) FTIR spectra of the extracted organic matrix after decalcification.

\subsection{Protein in the matrix}

It has become more and more clear that proteins play crucial roles during mineralization and assembly of marine shells. Proteins (small quantities of less than $1 \%$ ) along with the chitin network have been shown to provide structural integrity, functioning as soft, energy-dissipating matrix by hindering crack propagation through the interfaces and increasing the toughness (Feng et al., 2017; Zhang et al., 2012; Bram et al., 2012; Fabio and M., 2012). Therefore, we set to explore proteins extracted from the shell. Fig. 6A illustrates sodium dodecyl sulfate-polyacrylamide gel electrophoresis (SDS-PAGE) of the extracted proteins. Proteins were extracted after decalcification either using 1M EDTA or 5\% v/v acetic acid. Presence of more protein extracts was noticeable in the insoluble fractions than soluble fractions obtained independent of extraction method. Clear bands, one at $100 \mathrm{kD}$ and the other one at $45 \mathrm{kD}$ in the insoluble extracts were noticeable. However, we did not observe similar bands in the soluble fraction. Further, $100 \mathrm{kD}$ and $45 \mathrm{kD}$ were subjected to in-gel trypsin digest (Fig. 6B) for de novo sequencing. Ten fragments from tryptic digest of $100 \mathrm{kD}$ protein and six from $45 \mathrm{kD}$ were selected, which had the strongest peak as well as the highest quality factor. Fig. 6C and D illustrates product ion spectrum and identified peptides with de novo sequencing for the corresponding isolated tryptic fragments. To identify possible hits to existing protein sequences, resulted monoisotopic masses cross-referenced using MASCOT search engine (Matrix Science, London, UK; version 2.1) against NCBI server (http://www.ncbi.nlm.nih.gov). In addition to that, identified de novo sequences cross-checked manually against BLASTp, Swiss-Prot and UniProt. However, we did not find any significant hits. Even though it would be too early to draw any conclusion about the nature of the intact proteins using these identified short fragments, but analyzing the amino acid composition Fig. S3 showed $100 \mathrm{kD}$ protein might be rich in glycine, serine, asparagine, alanine, proline and arginine. However, $45 \mathrm{kD}$ proteins mostly contained alanine, glycine, glutamine, lysine, serine, tryptophan and tyrosine. 
(A)

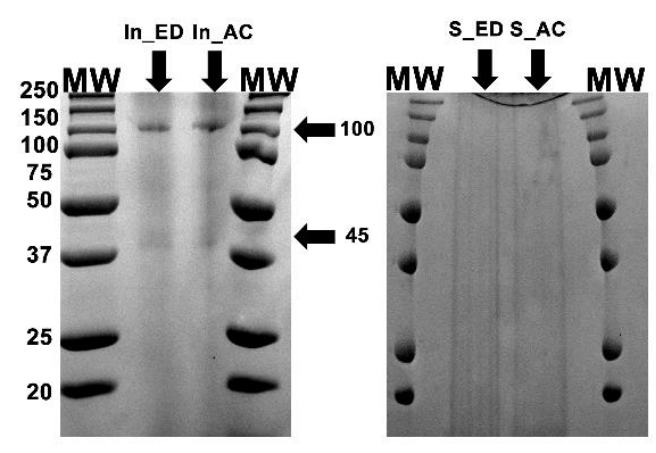

(B)

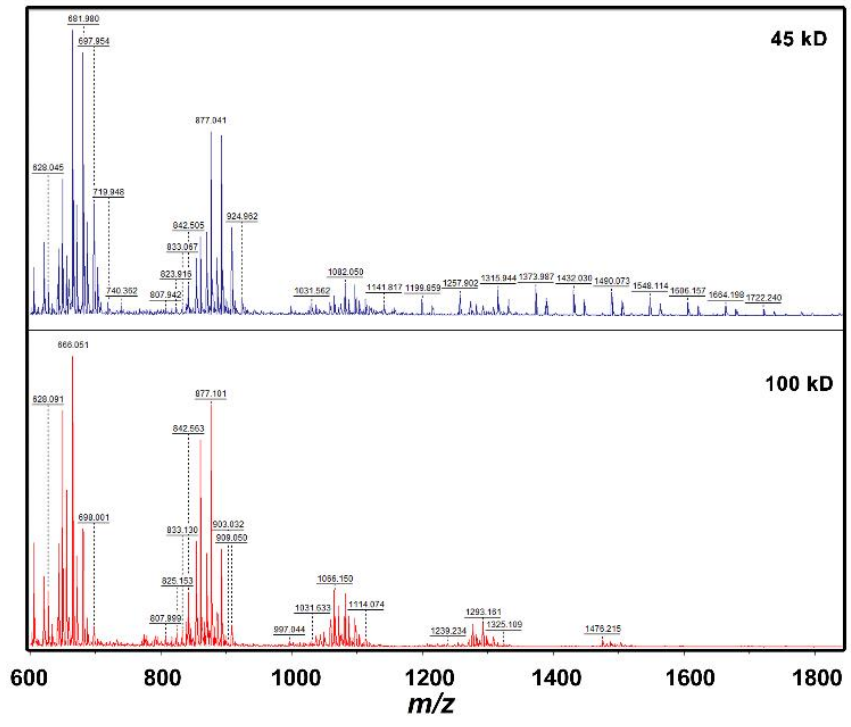

(C)

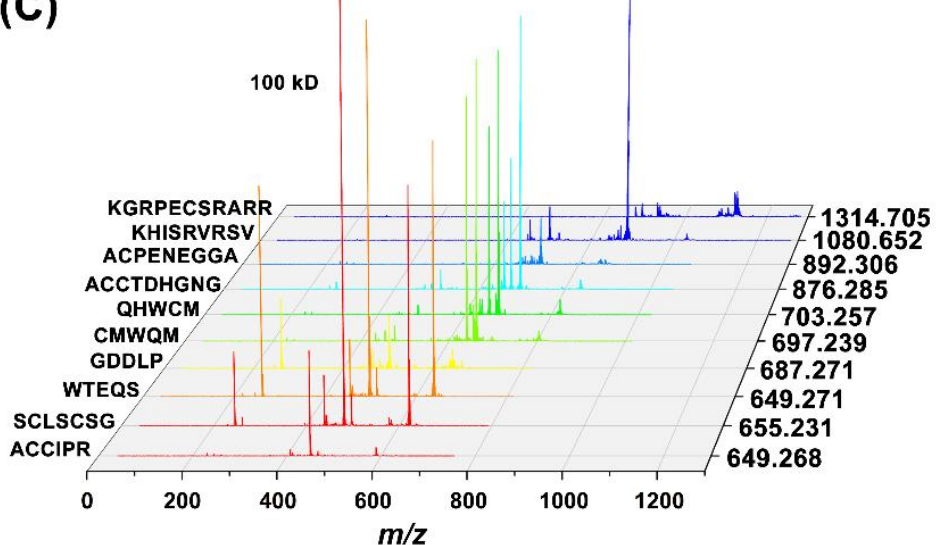

(D)

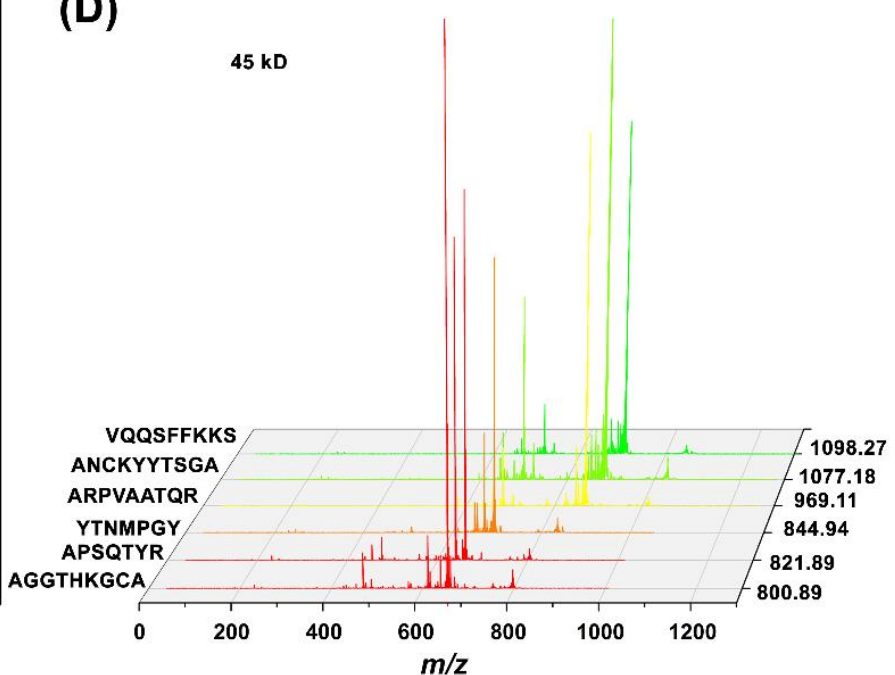

Fig. 6. (A) SDS-PAGE of the soluble and insoluble protein extracted after decalcification of the shell using $1 m$ EDTA pH 8 (marked as $S \_E D$ and In_ED) and 5\% v/v acetic acid (marked S_AC and In_AC). Molecular weight markers are indicated as MW. (B) Mass spectrum of fragments produced from tryptic digestion of $100 \mathrm{kD}$ (In_ED) and $45 \mathrm{kD}$ (In_ED) band. (C) MS/MS spectrum of ten peptides obtained from $100 \mathrm{kD}$ (IN-ED) digest with $\mathrm{m} / \mathrm{z}$ ranging from 650-1315 and their corresponding sequences. (D) MS/MS spectrum of six peptides from $45 \mathrm{kD}$ (IN-ED) digest with $\mathrm{m} / \mathrm{z}$ ranging from $800-1100$ and their corresponding sequences.

\section{Conclusion}

To our knowledge, this is the first detailed study on the structure of $M$. edulis L. shell, commonly known as Finnish blue mussel found in the archipelago of SW-Finland (Northern Baltic Sea). We employed various techniques to investigate in detail, both inorganic mineralized components as well as the organicrich matrix of M. edulis L. Being closely related to Mytilus edulis we found a very similar ultra- 
architecture across the shell. However, we also found differences, for instance in the thickness and degree of orientation of the layers. Using high resolution SEM imaging and probing the calcified rods in the oblique prismatic layer, we found pores with a diameters of 40-50 nm and lengths up to a micrometer extending through the rods. These channels were only found in the anterior ventral part which lacks the nacreous part. We also noted that the matrix material was highly fluid, oozing out from the cracked surfaces. The fluidity of the matrix and the presence of the channels in the more newly formed parts of the shell, leads us to suggest that the channels might have a role for transport of the fluid organic matrix. Our results also show that the shell exhibits a gradient of mechanical properties. Moving from anterior to posterior region, we noticed an increase of both stiffness and hardness. This likely attributes to the fact that each region has quite distinct morphology as they are in the different developmental stage. At the posterior region, the biomineralized layer grows and developed earlier, hence this resulted in thicker and more compact motifs with noticeable differences in the degree of orientation than the anterior region. We hypothesize that this could possibly be a generic characteristic for other closely related taxa of mussels including M. edulis as this has not been in the scope of earlier studies to our knowledge. This finding could provide inspiration for designing lightweight load-bearing materials in which a gradient of mechanical properties is crucial. There is growing evidence suggesting that the organic-rich interface largely governs both intrinsic and extrinsic toughening in such materials. In addition to that, their substantial importance for controlling both nucleation and inhibition of the crystal growth and the adhesion of the rod cannot be ignored. We identified chitin and protein as the main component of the organic-rich interfacial matrix. We found protein-chitin-rich interface forms less than $1 \%$ volume fraction of the shell. However sufficient to work in synergy with their stiff surrounding architecture to provide non-linear deformation upon initiation of the cracks. This results in the propagation of the cracks into conformations that requires considerable energy before undergoing catastrophic failure with elongated filaments of chitin-protein, bridging crack at the interface of the calcified rods. Proteomic analysis showed two abundant proteins with sizes around $100 \mathrm{kD}$ and $45 \mathrm{kD}$. In future, a combination of high-throughput RNA-sequencing, advanced proteomics analysis, and X-ray crystallography could be applied to identify the full-length sequences of these proteins and find possible sequence or structural homology to other known proteins. Eventually, this could be combined with molecular dynamic simulations to accelerate our understanding of their key molecular interactions with chitin and $\mathrm{CaCO}_{3}$ and their colloidal complexation toward structure formation. 


\section{Acknowledgments:}

We acknowledge Aalto University Nano-microscopy Center (Espoo) for their generous microscopy time. The work was performed within the Academy of Finland Center of Excellence Programme (2014-2019) and Academy of Finland projects 264493, 259034, and 317395. Chenghao Li is acknowledged for help during the synchrotron measurements at BESSY, Fabian Zemke for assistance with x-ray scattering data evaluation and Petra Leibner for performing the nanoindentation experiments.

\section{Author Contributions}

P.M and W.W designed and conducted the experiments. M.B.L supervised the project. All Authors contributed in writing the manuscript.

\section{Competing financial interests}

The authors declare no competing financial interest.

\section{References}

1. Ritchie, R. O. The conflicts between strength and toughness. Nat. Mater. 10, 817-822 (2011).

2. Ji, B. \& Gao, H. Mechanical properties of nanostructure of biological materials. J. Mech. Phys. Solids 52, 1963-1990 (2004).

3. Li, X. W., Ji, H. M., Yang, W., Zhang, G. P. \& Chen, D. L. Mechanical Properties of CrossedLamellar Structures in Biological Shells: A Review. J. Mech. Behav. Biomed. Mater. (2017).

4. Nudelman, F. et al. Forming nacreous layer of the shells of the bivalves Atrina rigida and Pinctada margaritifera: an environmental-and cryo-scanning electron microscopy study. $J$. Struct. Biol. 162, 290-300 (2008).

5. Ballarini, R. \& Heuer, A. H. Secrets in the Shell The body armor of the queen conch is much tougher than comparable synthetic materials. What secrets does it hold? Am. Sci. 95, 422-429 (2007).

6. Hou, D. F., Zhou, G. S. \& Zheng, M. Conch shell structure and its effect on mechanical behaviors. Biomaterials 25, 751-756 (2004).

7. Kamat, S., Su, X., Ballarini, R. \& Heuer, A. H. Structural basis for the fracture toughness of the shell of the conch Strombus gigas. Nature 405, 1036-1040 (2000).

8. Feng, D., Li, Q., Yu, H., Kong, L. \& Du, S. Identification of conserved proteins from diverse shell matrix proteome in Crassostrea gigas: characterization of genetic bases regulating shell formation. Sci. Rep. 7, 45754 (2017). 
9. Zhang, G. et al. The oyster genome reveals stress adaptation and complexity of shell formation. Nature 490, 49 (2012).

10. Fabio, N. \& M., S. N. A. J. Biomineralization as an Inspiration for Materials Chemistry. Angew. Chemie Int. Ed. 51, 6582-6596 (2012).

11. Bram, C. et al. Think Positive: Phase Separation Enables a Positively Charged Additive to Induce Dramatic Changes in Calcium Carbonate Morphology. Adv. Funct. Mater. 22, 907-915 (2012).

12. Studart, A. R. Additive manufacturing of biologically-inspired materials. Chem. Soc. Rev. 45, 359-376 (2016).

13. Wegst, U. G. K., Bai, H., Saiz, E., Tomsia, A. P. \& Ritchie, R. O. Bioinspired structural materials. Nat. Mater. 14, 23-36 (2015).

14. Studart, A. R. Towards high-performance bioinspired composites. Adv. Mater. 24, 5024-5044 (2012).

15. Yao, H., Ge, J., Mao, L., Yan, Y. \& Yu, S. 25th anniversary article: artificial carbonate nanocrystals and layered structural nanocomposites inspired by nacre: synthesis, fabrication and applications. Adv. Mater. 26, 163-188 (2014).

16. Mirkhalaf, M., Dastjerdi, A. K. \& Barthelat, F. Overcoming the brittleness of glass through bioinspiration and micro-architecture. Nat. Commun. 5, 3166 (2014).

17. Nudelman, F. \& Sommerdijk, N. A. J. M. Biomineralization as an inspiration for materials chemistry. Angew. Chemie Int. Ed. 51, 6582-6596 (2012).

18. Gu, G. X., Takaffoli, M. \& Buehler, M. J. Hierarchically enhanced impact resistance of bioinspired composites. Adv. Mater. 29, (2017).

19. Gao, H.-L. et al. Mass production of bulk artificial nacre with excellent mechanical properties. Nat. Commun. 8, 287 (2017).

20. Guerette, P. A. et al. Accelerating the design of biomimetic materials by integrating RNA-seq with proteomics and materials science. Nat. Biotechnol. 31, 908-915 (2013).

21. Benecke, G. et al. A customizable software for fast reduction and analysis of large X-ray scattering data sets: applications of the new DPDAK package to small-angle X-ray scattering and grazing-incidence small-angle X-ray scattering. J. Appl. Crystallogr. 47, 1797-1803 (2014).

22. Oliver, W. C. \& Pharr, G. M. An improved technique for determining hardness and elastic modulus using load and displacement sensing indentation experiments. J. Mater. Res. 7, 1564- 
1583 (1992).

23. Liu, C. et al. In-depth proteomic analysis of shell matrix proteins of Pinctada fucata. Sci. Rep. 5, 17269 (2015).

24. Vuorinen, I., Antsulevich, A. E. \& Maximovich, N. V. Spatial distribution and growth of the common mussel Mytilus edulis L. in the archipelago of SW-Finland, northern Baltic Sea. Boreal Environ. Res. 7, 41-52 (2002).

25. Feng, Q. L. et al. Crystallographic alignment of calcite prisms in the oblique prismatic layer of Mytilus edulis shell. J. Mater. Sci. 35, 3337-3340 (2000).

26. Marin, F., Le Roy, N. \& Marie, B. The formation and mineralization of mollusk shell. Front Biosci 4, 125 (2012).

27. Rahman, M. A. \& Halfar, J. First evidence of chitin in calcified coralline algae: new insights into the calcification process of Clathromorphum compactum. Sci. Rep. 4, 6162 (2014).

28. Weaver, J. C. et al. Analysis of an ultra hard magnetic biomineral in chiton radular teeth. Mater. Today 13, 42-52 (2010).

29. Kumirska, J. et al. Application of spectroscopic methods for structural analysis of chitin and chitosan. Mar. Drugs 8, 1567-1636 (2010). 